\title{
25143 Itokawa: direct detection of the current decelerating spin state due to YORP effect
}

\author{
K. Kitazato ${ }^{1,2}$, M. Abe $^{2}$, M. Ishiguro ${ }^{3}$, and W.-H. Ip ${ }^{4}$ \\ 1 Department of Earth and Planetary Science, The University of Tokyo, Bunkyo-ku, Tokyo 113-0033, Japan \\ e-mail: kitazato@planeta.sci.isas.jaxa.jp \\ 2 Institute of Space and Astronautical Science, Japan Aerospace Exploration Agency, Sagamihara, Kanagawa 229-8510, Japan \\ 3 School of Physics and Astronomy, Seoul National University, Seoul 151-747, Korea \\ ${ }^{4}$ Institute of Space Science, National Central University, 32054 Chung Li, Taiwan
}

Received 25 June 2007 / Accepted 9 July 2007

ABSTRACT

\begin{abstract}
Aims. The Hayabusa mission revealed fundamental physical properties of the small near-Earth asteroid 25143 Itokawa, such as shape and mass, during its rendezvous with the asteroid in 2005. Resulting from this, the YORP-induced change in the asteroid's spin state has been predicted theoretically. The purpose of this study is to investigate the YORP effect for Itokawa directly from an observational perspective.

Methods. A long-term campaign of ground-based photometric observations of Itokawa were performed from March 2001 to December 2006. The observed asteroid lightcurves were compared with numerical modeling using the detailed shape model and global surface photometric properties derived from the Hayabusa mission.

Results. As a non-linear time evolution of rotational phase lag is shown, we found that Itokawa has been decreasing its spin rate by $\dot{\omega}=-1.2( \pm 0.2) \times 10^{-17} \mathrm{rad} \mathrm{s}^{-2}$. The detected deceleration rate is almost consistent with, but slightly smaller than, the theoretically predicted value due to the YORP effect.
\end{abstract}

Key words. minor planets, asteroids - techniques: photometric

\section{Introduction}

The spin states of small asteroids can be secularly altered by a net torque arising from the anisotropic reflection and thermal re-emission of sunlight on the surface, the so-called Yarkovsky-O'Keefe-Radzievskii-Paddack (YORP) effect, in addition to abrupt torques due to tidal encounters and impacts. Such radiation torques are a significant perturbation, especially for near-Earth asteroids in close proximity to the Sun, and have been invoked as a possible contributor in creating binary asteroids and fast rotators. Indirect evidence of the YORP effect has been found in the preferential alignment of spin axes in the Koronis asteroid family (Silvan 2002; Vokrouhlický et al. 2003). Recently, spin rate variations were detected directly from ground-based astronomical techniques for two near-Earth asteroids: 1862 Apollo (Kaasalainen et al. 2007), and 54509 YORP $\left(2000 \mathrm{PH}_{5}\right)$ (Lowry et al. 2007; Taylor et al. 2007).

The near-Earth asteroid 25143 Itokawa has a size of roughly $300 \mathrm{~m}$ in diameter and an irregularly shaped body. Its fundamental parameters were precisely determined through the proximity observations of Hayabusa spacecraft in 2005 (Fujiwara et al. 2006). Vokrouhlický et al. (2004) theoretically estimated the change in rotation rate due to the YORP effect for Itokawa using pre-rendezvous shape models. More recently, Scheeres et al. (2007) refined the computation of the YORP effect using the detailed shape model, rotation pole, mass estimate, and optical properties derived from the Hayabusa mission, predicting a large deceleration that should be detectable over time scales as short as several years. However, in the previous studies of groundbased photometric observations in 2001-2004, the rotation rate variations for the asteroid were not discernible beyond uncertainties of photometry and shape determination (Kaasalainen et al. 2003, 2004).

Here we present the results of independent photometric observations of Itokawa from March 2001 to December 2006. The observed lightcurves are compared with the corresponding modeled ones using the shape model and photometric properties derived from the Hayabusa mission.

\section{Observations and analyses}

Time-resolved photometric observations provide information on the spin state of the asteroid. The photometric observations of Itokawa were started in March 2001, and were performed on a total of 27 nights for 7 apparitions until December 2006, with three telescopes. A list of the telescopes and instrumentation used in this study is presented in Table 1. The geometric circumstances are summarized in Table 2. This table gives the mid-time of observation coverage on a day, the geocentric ecliptic longitudes $(\lambda)$ and latitudes $(\beta)$ of the asteroid (for equinox J2000), its heliocentric $(r)$ and geocentric $(\Delta)$ distances, the solar phase angle $(\alpha)$, and the observatory number from Table 1 . The observations of the asteroid were taken in the Cousins $R$ filter while tracking at the asteroid's predicted ephemeris rate, where exposure times were set to within the range of 60 to $600 \mathrm{~s}$. The observing setup and the data reduction were carried out by a consistent method among all different observatories.

The CCD image pre-reductions were made with standard IRAF routines, removing bias and dark current from the images. 
Table 1. Telescopes and instrumentation.

\begin{tabular}{lll}
\hline \hline & Telescope & Instrument \\
\hline 1 & Kiso Observatory, 1.05-m & SITe 2k $\times 2 \mathrm{k}$ \\
2 & Univ. Hawaii on Mauna Kea, 2.2-m & 8k $\times 8 \mathrm{k}$ CCD \\
3 & Lulin Observatory, 1-m & VersArray: 1300B \\
\hline
\end{tabular}

Table 2. Geometric circumstances for 25143 Itokawa.

\begin{tabular}{llcccccc}
\hline \hline Date UT & & $\begin{array}{c}\lambda \\
\left({ }^{\circ}\right)\end{array}$ & \multicolumn{1}{c}{$\begin{array}{c}r \\
\left({ }^{\circ}\right)\end{array}$} & $\begin{array}{c}r \\
(\mathrm{AU})\end{array}$ & $\begin{array}{c}\Delta \\
(\mathrm{AU})\end{array}$ & $\begin{array}{c}\alpha \\
\left({ }^{\circ}\right)\end{array}$ & Obs. $^{a}$ \\
\hline 2001 Mar. & 26.7 & 236.3 & 43.1 & 1.02 & 0.04 & 60.2 & 1 \\
& 29.7 & 260.3 & 43.6 & 1.01 & 0.04 & 74.4 & 1 \\
& 31.7 & 275.9 & 41.2 & 1.00 & 0.04 & 83.9 & 1 \\
2001 Apr. & 1.7 & 282.2 & 39.4 & 1.00 & 0.04 & 88.3 & 1 \\
& 22.7 & 355.0 & -7.1 & 1.31 & 0.32 & 20.1 & 1 \\
& 23.6 & 354.5 & -7.0 & 1.31 & 0.32 & 19.0 & 1 \\
& 24.7 & 353.9 & -7.0 & 1.31 & 0.32 & 17.8 & 1 \\
& 25.6 & 353.3 & -7.0 & 1.32 & 0.33 & 16.7 & 1 \\
& 1.7 & 130.2 & 1.4 & 1.53 & 0.78 & 34.5 & 1 \\
& 2.7 & 130.5 & 1.5 & 1.52 & 0.77 & 34.2 & 1 \\
& 3.7 & 130.7 & 1.5 & 1.52 & 0.76 & 34.0 & 1 \\
& 4.7 & 131.0 & 1.5 & 1.52 & 0.75 & 33.8 & 1 \\
& 15.6 & 129.8 & 4.5 & 1.37 & 0.40 & 11.2 & 1 \\
& 19.7 & 128.2 & 4.8 & 1.35 & 0.37 & 7.6 & 1 \\
& 20.6 & 127.8 & 4.9 & 1.35 & 0.37 & 6.8 & 1 \\
& 10.5 & 107.6 & 6.3 & 1.02 & 0.25 & 79.2 & 1 \\
& 11.5 & 107.9 & 6.3 & 1.02 & 0.25 & 80.0 & 1 \\
& 12.5 & 108.2 & 6.3 & 1.01 & 0.25 & 80.7 & 1 \\
& 6.3 & 308.2 & -6.1 & 1.30 & 0.35 & 27.2 & 2 \\
& 7.3 & 308.4 & -6.0 & 1.31 & 0.35 & 27.6 & 2 \\
& 8.3 & 308.6 & -5.9 & 1.31 & 0.36 & 28.0 & 2 \\
& 9.3 & 308.8 & -5.8 & 1.32 & 0.37 & 28.4 & 2 \\
& 18.7 & 112.0 & 1.9 & 1.53 & 0.58 & 16.0 & 3 \\
& 19.5 & 111.7 & 2.0 & 1.53 & 0.57 & 15.4 & 3 \\
& 20.5 & 111.4 & 2.0 & 1.52 & 0.57 & 14.6 & 3 \\
& 21.6 & 111.0 & 2.1 & 1.52 & 0.56 & 13.7 & 3 \\
& 22.6 & 110.7 & 2.1 & 1.52 & 0.55 & 12.9 & 3 \\
\hline
\end{tabular}

${ }^{a}$ See Table 1 for telescopes and instrumentation.

Nightly flat-field frames were used to correct images for optical vignetting, dust shadow and pixel-to-pixel sensitivity variation. The images were reduced with the aperture photometry. A large number of local comparison stars (typically more than ten) were selected along the asteroid path. From this set of comparison stars, the non-variable stars with small error in the flux measurements were chosen as reference stars for the lightcurve. The atmospheric extinction coefficient was determined nightly through the Landolt standard stars (Landolt 1992). The individual lightcurves were corrected for light-travel time and the magnitudes were reduced to unit geocentric and heliocentric distances of the asteroid.

\section{Numerical modeling of lightcurves}

To generate synthetic lightcurves for each observing time, we used the most up-to-date Itokawa shape model consisting of 49, 152 facets (Gaskell et al. 2006), which was developed based on the remote-sensing images taken by the Hayabusa onboard camera. The brightness of the asteroid as seen by the Earth observer is computed as a sum of contributions from all visible facets, using ray-tracing methods that check which facets are occulted by or in shadow from the other facets, where the surface scattering property is given by Hapke's bidirectional reflectance model (Hapke 1993, 2002). We assume the following values of globally-averaged photometric parameters in the Hapke model, based on the photometry results of Itokawa from the Hayabusa near-infrared spectrometer (Kitazato et al. 2007): the single-particle scattering albedo $\omega=0.4$, the asymmetry parameter of single-particle phase function $g=-0.35$, opposition surge amplitude $B_{0}=0.89$ and width $h=0.01$, and macroscopic roughness $\bar{\theta}=26^{\circ}$.

Since quite a small change in the obliquity of Itokawa for several years is predicted for its YORP effect (Scheeres et al. 2007), it is assumed that the spin axis remains constant in our calculations. The rotation period has been estimated to be (12.13237 \pm 0.00008$)$ hours in Kaasalainen et al. (2004), and the epoch of prime meridian corresponding to the celestial reference frame has been well constrained within a small error (less than 0.001 degree as phase lag in the asteroid rotation) among the Hayabusa rendezvous phase (Gaskell et al. 2006). Thus, we use a constant sidereal rotation period of $P=12.13237$ hour and a zero-phase Julian day epoch of $t_{0}=2453644.5$ (1.0 October 2005).

\section{Comparing between the observed and modeled lightcurves}

The observed lightcurve points and the corresponding modeled lightcurves in the representative apparitions are shown in Fig. 1. In our numerical simulations of the asteroid lightcurve the rotational period is set at a constant value. Hence, if the asteroid's spin rate varies in practice, it can be considered that a certain phase lag would occur between the observed and modeled lightcurves. There is little difference between the lightcurve shapes, although a slight discrepancy in absolute brightness was found due to uncertainties of the photometric calibrations. The given uncertainty of pole orientation is small enough not to alter the profile of simulated lightcurves. When sufficiently goodquality data covering over one or more lightcurve maximum or minimum are available, we can determine the phase lag along with the vertical shift on each dataset. Figure 2 shows an enlarged view of the lightcurves in the 2001 March apparition, when they are furthest from the zero-phase epoch as defined above. In this figure, the best-fit synthetic lightcurves are also plotted, and these fitted curves systematically shift in the same direction as against the original curves.

All the values of phase lag estimated in each observed lightcurve data are shown in Fig. 3, where errors of the phase lags are given by one standard deviation between the observed lightcurve points and the best-fitted simulated lightcurve. Here, it is found that these phase lag values stay within the confines predicted by uncertainties in rotation period (maximum and minimum rotation period). Thus, our results are consistent with the previous analyses of Kaasalainen et al. (2004). In addition, we found that Itokawa's spin behavior could not be explained with a constant rotation period, as the phase lag changes with nonlinearity. To achieve a data fit, we consider a simple model in which the asteroid spin velocity increases linearly in time $t$. The time evolution of the phase lag is described as

$\Delta \phi=2 \pi \frac{\Delta P}{P_{0}^{2}}\left(t-t_{0}\right)+\frac{1}{2} \dot{\omega}\left(t-t_{0}\right)^{2}$, 

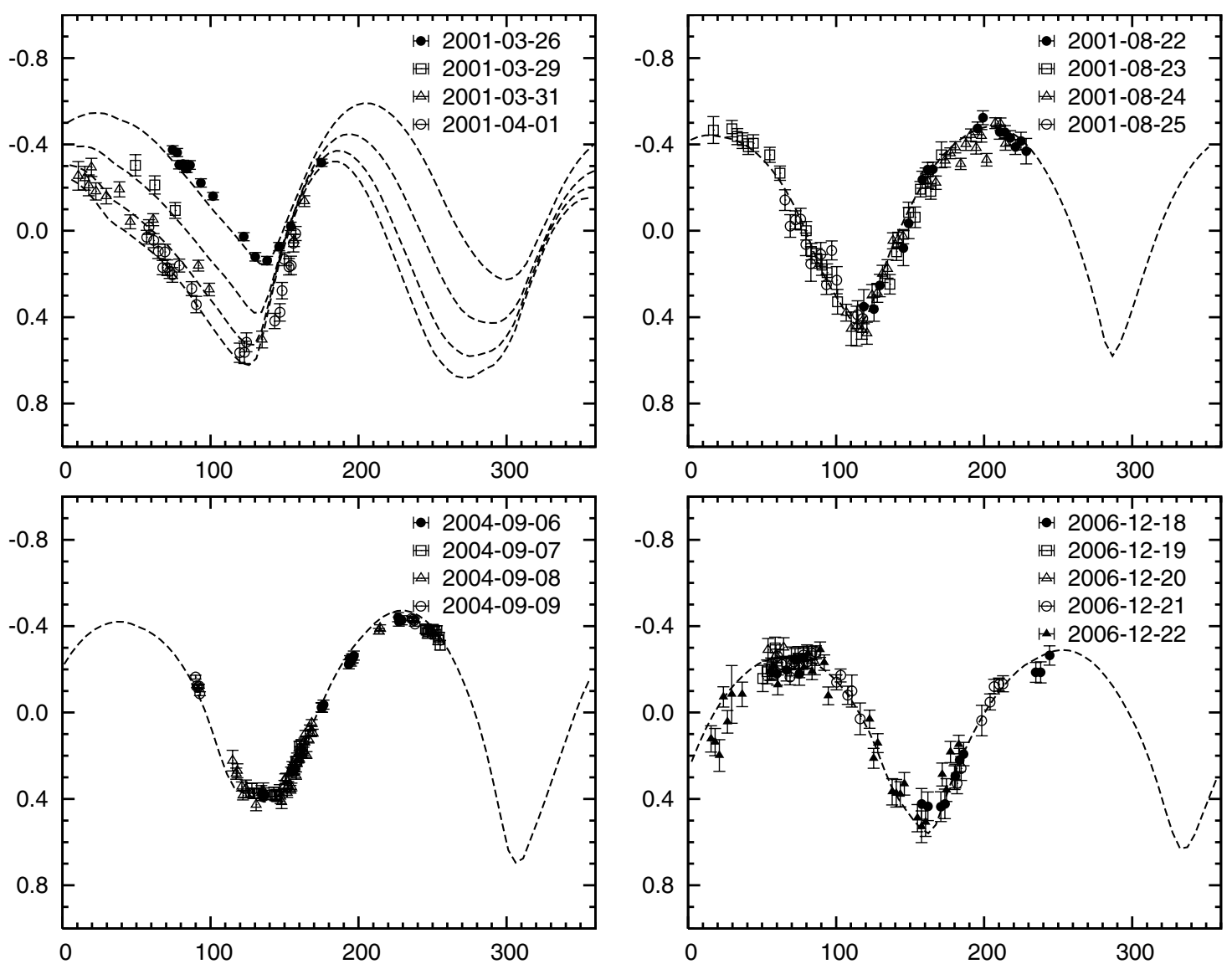

Fig. 1. The representative observed data (points) and the corresponding modeled lightcurves (dashed lines) for the asteroid 25143 Itokawa, added the arbitrary intensity offset for clarity. The horizontal axes give the rotational phase in degrees, and the vertical axes brightness in units of relative intensity. For each of the apparitions in August 2001, September 2004 and December 2006, only one modeled curve has been plotted.

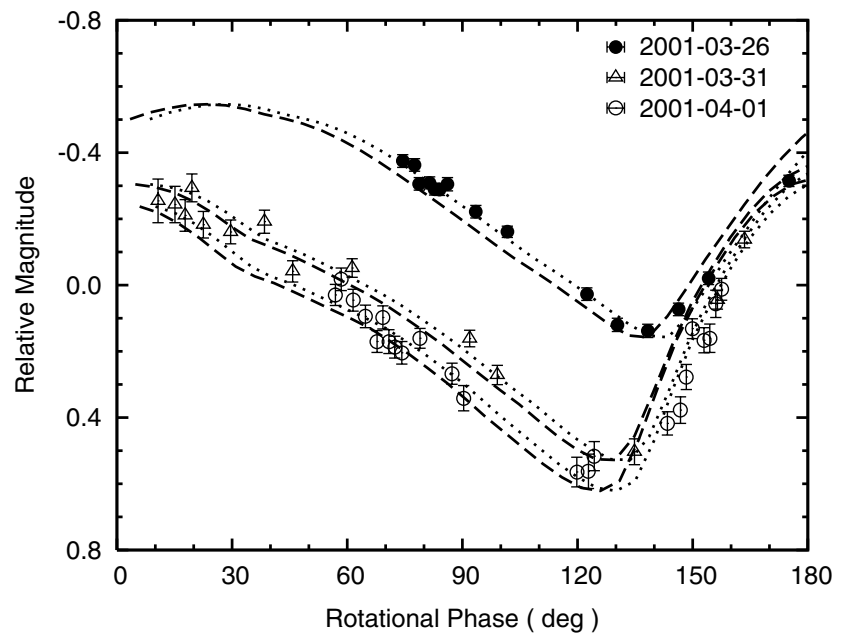

Fig. 2. Enlarged view of the lightcurves in the 2001 March apparition are shown, together with the best-fit synthetic lightcurves based on phase lag against the observed data (dotted lines). Data for 2001 March 29 are removed here due to lack of coverage enough to fit.

where $\Delta P$ is the time offset toward the nominal rotation period and $\dot{\omega}$ is the angular acceleration. From a non-linear least square fitting with this expression, the best-fit solutions were determined by $\dot{\omega}=-1.2( \pm 0.2) \times 10^{-17} \mathrm{rad} \mathrm{s}^{-2}$ and $\Delta P=0.09 \pm 0.07 \mathrm{~s}$, indicating that the asteroid's spin rate has been decreasing. A constant-period fit yields a weighted sum of squared residuals $30 \%$ higher than that for the solution with acceleration. As a result, the more precise rotation period for Itokawa is provided as $P_{0}=12.132395 \pm 0.000019$ h on 1.0 October 2005 .

\section{Discussions}

As another major mechanism to modify an asteroid's spin state, tidal torques during close approaches to planets should also be included as well as YORP torques. According to Vokrouhlický et al. (2004), the effect of tidal torque for Itokawa is small compared to YORP, though not negligible. The expected YORP behavior is essentially a linear secular trend in time, with only small fluctuations owing to orbital dynamics around the Sun. Therefore, almost all the spin rate change of Itokawa detected in this study can be accounted for by YORP. Comparing to the theoretically predicted amount of Itokawa's YORP from Scheeres et al. (2007), we find that our measurement is consistent in terms of decelerating, but the deceleration rate is marginally below the range predicted (Table 3). The observed lower amount implies that the YORP effect partially depends on a finer scale of surface roughness than present in the resolutions of the derived shape models.

The observed YORP amounts for the near-Earth asteroids 1862 Apollo and 54509 YORP are presented in Table 3, where the normalized acceleration rate $\dot{\omega}_{\text {norm }}$ is the value with semimajor axis $a=1 \mathrm{AU}$ and equivalent size $D=1.0 \mathrm{~km}$. Note that Apollo and YORP are spun up while Itokawa is spun down. The spin rate change due to YORP for Itokawa is smaller than those for the other near-Earth asteroids, Apollo and YORP. It seems easy to explain such differences by their surface 


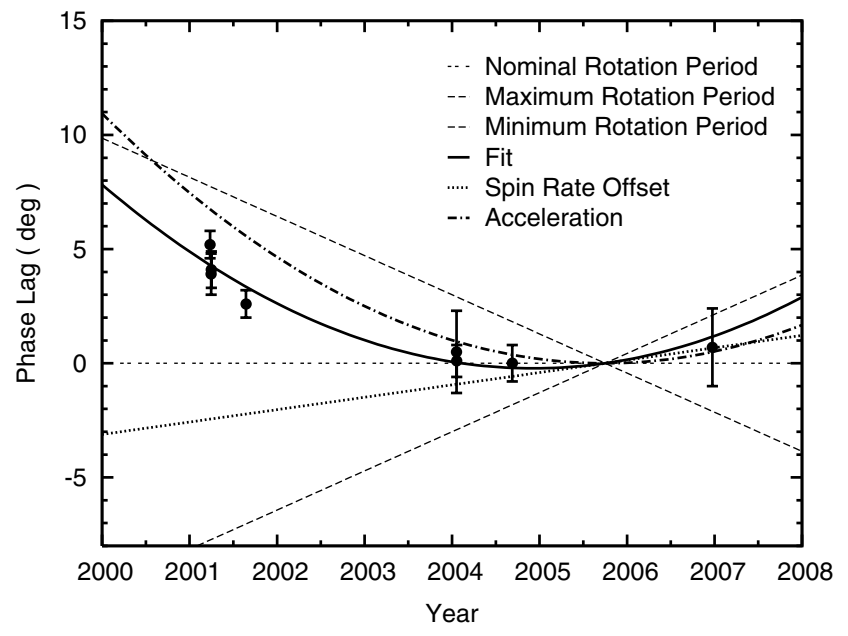

Fig. 3. Variations of the rotational phase lag between the observed and modeled lightcurves as a function of time (close circles). The lines display the relative phase change linearly due to uncertainty in the rotation period (maximum and minimum) from Kaasalainen et al. (2004), and the best-fit model curve using Eq. (1) and its components of spin rate offset and acceleration, respectively.

Table 3. Normalized acceleration/deceleration rate for three near-Earth asteroids.

\begin{tabular}{lcl}
\hline \hline Object & $\begin{array}{c}\dot{\omega}_{\text {norm }} \\
\left(10^{-18} \mathrm{rad} \mathrm{s}^{-2}\right)\end{array}$ & References \\
\hline Itokawa (obs) & -2.2 & This study \\
Itokawa (model) & $-8.2 \sim-4.6$ & Scheeres et al. (2007) \\
Apollo & 30 & Kaasalainen et al. (2007) \\
YORP (2000 $\left.\mathrm{PH}_{5}\right)$ & 6.2 & Lowry et al. (2007) \\
\hline
\end{tabular}

topographies and obliquities rather than the densities and surface thermal properties because of the similarity of taxonomic types between Itokawa and Apollo, S-type and Q-type, respectively (Binzel et al. 2001; Tholen \& Barucci 1989). The comparison of theoretical computations of YORP spin accelerations with measured accelerations for 54509 YORP in Taylor et al. (2007) differ by a similar factor as mentioned above for Itokawa. This may point out some systematic deficiency in current YORP theoretical models.

Moreover, the observed deceleration rate indicates that Itokawa had been rotating faster in the past. As discussed in Scheeres et al. (2007), it is probable that the mutual orbiting system of the two components, the "head" and "body" of Itokawa, would be bound when the rotation period approaches an approximate value of $6.5 \mathrm{~h}$. Using our results, it could be estimated that such an event occurred in approximately $0.4 \mathrm{Ma}$. This timescale is considerably shorter than the near-Earth asteroid's lifetime ( 10 My).

\section{Summary}

A YORP-induced decelerating spin state for Itokawa was detected by the comparison between its observed and modeled lightcurves. Thanks to the detailed shape model derived from the Hayabusa mission, the time evolution of the lightcurve phase lag could be determined more accurately than the previous studies without uncertainty of the asteroid shape and pole orientation. Itokawa has been decreasing its rotation rate by $\dot{\omega}=$ $-1.2( \pm 0.2) \times 10^{-17} \mathrm{rad} \mathrm{s}^{-2}$, the normalized amount of which is smaller than those of the other YORP-detected near-Earth asteroids, Apollo and YORP.

Acknowledgements. The authors thank all the staffs at the observatories for their support in performing this research and are grateful to S. Nishihara, Y. Ohba, Y. Sarugaku and D. Kinoshita for their assistance to our observations and analyses. K.K. and M.A. acknowledge support from the Research Fellowships of the Japan Society for the Promotion of Science for Young Scientists and the Grant-in-Aid for Scientific Research (B) 17340133, respectively. The work of W.H.I has been supported by NSC in Taiwan.

\section{References}

Binzel, R. P., Rivkin, A. S., Bus, S. J., et al. 2001, Meteorit. Planet. Sci., 36, 1167 Fujiwara, A., Kawaguchi, J., Yeomans, D. K., et al. 2006, Science, 312, 1330

Gaskell, R. W., Barnouin-Jha, O. S., Scheeres, D., et al. 2006, Landmark navigation studies and target characterization in the Hayabusa encounter with Itokawa. AIAA paper 2006 6660, AAS/AIAA Astrodynamics Specialists Conf., Keystone, CO, Aug.

Hapke, B. 1993, Theory of Reflectance and Emittance Spectroscopy (Cambridge, UK: Univ. Press, Cambridge)

Hapke, B. 2002, Icarus, 157, 523

Kaasalainen, M., Kwiatkowski, T., Abe, M., et al. 2003, A\&A, 405, L29

Kaasalainen, M., Abe, M., Byron, J., et al. 2004, 2001-2004 and modeling of asteroid (25143) Itokawa, Int. Sci. Symp. Sample Returns from Solar System Minor Bodies, Abstract

Kaasalainen, M., Durech, J., Warner, B. D., et al. 2007, Nature, 446, 420

Kitazato, K., Clark, B. E., Abe, M., et al. 2007, Icarus, submitted

Landolt, A. U. 1992, AJ, 104, 340

Lowry, S. C., Fitzsimmons, A., Pravec, P., et al. 2007, Science, 316, 272

Scheeres, D. J., Abe, M., Yoshikawa, M., et al. 2007, Icarus, 188, 425

Silvan, S. M. 2002, Nature, 419, 49

Taylor, P. A., Margot, J. L., Vokrouhlický, D., et al. 2007, Science, 316, 274

Tholen, D. J., Barucci, M. A. 1989, in Asteroids II, ed. R. P. Binzel, T. Gehrels, \& M. S. Matthews (Tucson: Univ. of Arizona Press), 298

Vokrouhlický, D., Nesvorný, \& D., Bottke, W. F. 2003, Nature, 425, 147

Vokrouhlický, D., Čapek, D., Kaasalainen, M., et al. 2004, A\&A, 414, L21 